\title{
Glucagon, Catecholamine and Pancreatic Polypeptide Secretion in Type I Diabetic Recipients of Pancreas Allografts
}

\author{
Peter Diem, * J. Bruce Redmon, * Munir Abid, * Antoinette Moran, ${ }^{\circ}$ David E. R. Sutherland, \\ Jeffrey B. Halter," and R. Paul Robertson* \\ ${ }^{*}$ Diabetes Center and Division of Endocrinology, Department of Medicine, ${ }^{\ddagger}$ Department of Pediatrics, ${ }^{\S}$ Department of Surgery, \\ University of Minnesota, Minneapolis, Minnesota; and "Department of Medicine, University of Michigan and the Veterans \\ Administration Medical Center, Ann Arbor, Michigan
}

\section{Abstract}

Successful pancreas transplantation in type I diabetic patients restores normal fasting glucose levels and biphasic insulin responses to glucose. However, virtually no data from pancreas recipients are available relative to other islet hormonal responses or hormonal counterregulation of hypoglycemia. Consequently, glucose, glucagon, catecholamine, and pancreatic polypeptide responses to insulin-induced hypoglycemia and to stimulation with arginine and secretin were examined in 38 diabetic pancreas recipients, 54 type I diabetic nonrecipients, and 26 nondiabetic normal control subjects. Glucose recovery after insulin-induced hypoglycemia in pancreas recipients was significantly improved. Basal glucagon levels were signifcantly higher in recipients compared with nonrecipients and normal subjects. Glucagon responses to insulin-induced hypoglycemia were significantly greater in the pancreas recipients compared with nonrecipients and similar to that observed in control subjects. Glucagon responses to intravenous arginine were significantly greater in pancreas recipients than that observed in both the nonrecipients and normal subjects. No differences were observed in epinephrine responses during insulin-induced hypoglycemia. No differences in pancreatic polypeptide responses to hypoglycemia were observed when comparing the recipient and nonrecipient groups, both of which were less than that observed in the control subjects. Our data demonstrate significant improvement in glucose recovery after hypoglycemia which was associated with improved glucagon secretion in type I diabetic recipients of pancreas transplantation. (J. Clin. Invest. 1990. 86:2008-2013.) Key words: pancreas transplantation • glucagon • catecholamines • pancreatic polypeptide

Presented in part at the 101st Annual Meeting of the Association of American Physicians, Washington, May 1988, the 48th Annual Meeting of the American Diabetes Association, New Orleans, June 1988, and at the 13th International Diabetes Federation Congress, Sydney, Australia, November 1988.

Address correspondence and reprint requests to Dr. R. Paul Robertson, The Diabetes Center, Box 101 UMHC, University of Minnesota, Minneapolis, MN 55455.

Received for publication 26 February 1990 and in revised form 13 July 1990.

J. Clin. Invest.

(c) The American Society for Clinical Investigation, Inc. 0021-9738/90/12/2008/06 \$2.00

Volume 86, December 1990, 2008-2013

\section{Introduction}

After successful pancreas transplantation, recipients with type I diabetes mellitus achieve a return to normal fasting plasma glucose levels, normal oral glucose tolerance tests, and normal levels of $\mathrm{HbA}_{1} \mathrm{C}(1-7)$. However, whether recipients undergo alterations in counterregulatory hormonal responses to glycopenia, or improvement in counterregulation of hypoglycemia, has not been determined. These are important considerations because type I diabetic patients commonly have defective glucagon and epinephrine responses to hypoglycemia and, consequently, deranged counterregulation of hypoglycemia with its attendant risks (8-13). Therefore, presence or absence of improvement in these responses are highly relevant factors in the evaluation of the overall benefits of pancreas transplantation. Moreover, since the transplanted pancreas is denervated and since islet alpha cell and pancreatic polypeptide cell function is neurally modulated, investigation of pancreas recipients offers a unique opportunity to assess regulation of glucagon and pancreatic polypeptide secretion from the pancreas in humans in the setting of absent central nervous system input into the islet.

To evaluate hormonal counterregulation of hypoglycemia in patients with type I diabetes mellitus who have received successful pancreas allografts, we have examined glucose, glucagon, pancreatic polypeptide, and catecholamine responses during insulin-induced hypoglycemia. We have also assessed glucagon and pancreatic polypeptide responsivity to direct stimulators of alpha and pancreatic polypeptide cells (arginine and secretin, respectively) to ascertain whether possible alterations in secretory responses in pancreas recipients are specific for hypoglycemic signaling or more general in nature. In this study, the responses of pancreas recipients were compared with responses of patients with similar durations of type I diabetes mellitus, who had not received pancreas transplantation, and to nondiabetic control subjects.

\section{Methods}

Subjects. 38 type I diabetic recipients of pancreas allografts with iliac vessel anastomoses and with intact native pancreases who had been transplanted between 3 and 60 mo previously were studied (Table I). All recipients were normoglycemic, had normal hemoglobin $A_{1} C$ levels and mildly elevated serum creatinine levels, and none were receiving exogenous insulin or other medication for diabetes. Immunosuppression was achieved with the triple-drug regimen of azathioprine, cyclosporine, and prednisone (14). 54 patients with type I diabetes and with similar age, sex distribution, body-mass index, serum creatinine levels, and duration of diabetes, as well as 26 normal volunteers of similar age, sex distribution, and body-mass index, were studied to obtain control data. All subjects were admitted to the University 
Table I. Characteristics of Type I Diabetic Recipients of Pancreas Allografts, Type I Diabetic Nonrecipients, and Normal Control Subjects

\begin{tabular}{lccc}
\hline & Recipients & Nonrecipients & Controls \\
\hline$n$ & 38 & 54 & 26 \\
Age $(y)$ & $34 \pm 6$ & $33 \pm 8$ & $34 \pm 14$ \\
Duration of diabetes & & & \\
$\quad$ mellitus $(y)$ & $22 \pm 6$ & $22 \pm 7$ & \\
$\mathrm{BMI}^{2}\left(\mathrm{~kg} / \mathrm{m}^{2}\right)$ & $23.4 \pm 3.7$ & $23.5 \pm 3.0$ & $23.0 \pm 3.1$ \\
HbA 1 Ic $(\%)$ & $5.2 \pm 0.7$ & $9.0 \pm 1.6$ & $5.2 \pm 0.5$ \\
Serum creatinine & & & \\
$\quad(\mu m o l / l i t e r)$ & $136 \pm 7$ & $162 \pm 27$ & $77 \pm 3$ \\
Duration after pancreas & $22 \pm 6$ & & \\
$\quad$ transplantation $(m o)$ & (range: $3-60)$ & & \\
& & & \\
\hline
\end{tabular}

Twelve of the diabetic subjects were studied before and after pancreas transplantation. All data are expressed as mean \pm 1 SD.

of Minnesota Clinical Research Center and tests were begun at 8:00 a.m. with the subjects at bedrest after fasting overnight. Informed written consent was obtained from all subjects and the test procedures had been approved by the University of Minnesota Human Subjects Committee. All tests were not performed on all subjects.

Insulin-induced hypoglycemia. 24 type I diabetic recipients, 48 type I diabetic nonrecipients, and 16 normal control subjects underwent insulin tolerance tests. An intravenous line was placed in an antecubital vein to draw samples for glucose, glucagon, pancreatic polypeptide, and catecholamines. In type I diabetic nonrecipients normoglycemia was first established with an intravenous insulin infusion for 6-12 h overnight. Two baseline samples were withdrawn during normoglycemia 5 and 0 min before insulin was injected intravenously to induce hypoglycemia. Pancreas recipients and nonrecipients were given $0.15-0.2 \mathrm{U} / \mathrm{kg}$, whereas controls were given $0.05 \mathrm{U} / \mathrm{kg}$ of insulin as an intravenous pulse to achieve similar glucose nadirs in all groups. Additional blood samples were withdrawn at $5,10,15,20,25,30,35,40$, 45,50 , and $60 \mathrm{~min}$ after the insulin pulse. Patients who experienced extreme drowsiness and/or who complained of severe discomfort received $25 \mathrm{~g}$ of intravenous glucose to terminate hypoglycemia.

Arginine and secretin stimulation. To compare glucagon responses induced by hypoglycemia with responses induced by a more directly acting secretagogue, 25 type I diabetic recipients, 47 type I diabetic nonrecipients, and 18 normal control subjects were given a maximally effective pulse of arginine ( $5 \mathrm{~g}$ in $25 \mathrm{ml}$ of water IV). To compare pancreatic polypeptide responses to hypoglycemia with a more directly acting agonist, 25 recipients, 17 type I diabetic nonrecipients, and 17 normal control subjects responses received a maximally effective pulse of secretin $(2 \mathrm{U} / \mathrm{kg}$ body weight in $0.9 \% \mathrm{NaCl}$ IV). Three baseline samples and additional samples 2, 3, 4, 5, 7, and 10 min after the respective injections were withdrawn for determination of glucagon or hPP levels, respectively.

Analytical methods. Serum glucose was immediately measured with a Beckman Glucose Analyzer II (Beckman Instruments Inc., Fullerton, CA). Samples for glucagon determinations were collected into prechilled tubes containing $2.5 \mathrm{mg}$ EDTA and $500 \mathrm{U}$ Trasylol per milliliter blood, put on ice, and centrifuged immediately. Glucagon was measured by radioimmunoassay (15) with antibody $04 \mathrm{~A}$ obtained from Dr. R. H. Unger (University of Texas, Dallas). Pancreatic polypeptide was measured by radioimmunoassay (16) according to Chance et al. using antibody kindly provided by Dr. R. E. Chance (Ely Lilly and Co., Indianapolis, IN). Catecholamines were measured using a radioenzymatic method described previously (17).

Data analysis. Data are expressed as mean \pm SEM unless otherwise stated. Statistics for intergroup comparisons were performed by analysis of variance followed by Fisher's least significant difference. When applicable, Student's paired or unpaired $t$ test were used. Discrete variables were compared by chi-squares analysis. Least-squares linear regression was used to assess bivariate interdependency. All $P$ values were for two-tailed distributions and $P<0.05$ was considered statistically significant.

\section{Results}

Glucose suppression and recovery during insulin tolerance testing. Similar blood glucose nadirs were obtained in all three groups $(1.8 \pm 0.1 \mathrm{mM}$ in recipients, $2.0 \pm 0.1 \mathrm{mM}$ in nonrecipients, and $2.0 \pm 0.1 \mathrm{mM}$ in controls; $P=\mathrm{NS}$ ). However, the fall in glucose tended to be somewhat slower in the nonrecipients than in the two other groups (Fig. 1). Blood glucose recovery after insulin-induced hypoglycemia in diabetic nonrecipients was severely impaired, the maximal blood glucose recovery being only $8 \pm 2 \%$ of decrement. Blood glucose recovery was significantly better in recipients $(34 \pm 4 \%$ of decrement; $P$ $<0.0001)$ but significantly lower than that observed in controls $(58 \pm 3 \%$ of decrement; $P<0.0001)$. All normal subjects and diabetic patients reached glucose levels below $2.6 \mathrm{mM}$ and all completed at least $35 \mathrm{~min}$ of this test from the time of insulin injection. Only 2 of 24 recipients could not complete the test because of symptoms of hypoglycemia. In contrast, the test was prematurely terminated by giving intravenous glucose because of marked symptoms of hypoglycemia in 19 out of 48 nonrecipients $(P<0.01)$. Consequently, the mean data illustrated in Figs. 1, 5, and 6 most likely result in an overestimation of blood glucose recovery and of hormonal responses during the last third of the test in the nonrecipient group because only those nonrecipients with comparatively less impaired hormonal responses were able to complete the test. In a subset of 12 type I diabetic patients who were studied before and after pancreas transplantation, blood glucose recovery after insulin-induced hypoglycemia improved in each individual and the difference in blood glucose recovery was highly significant ( $13 \pm 6 \%$ vs. $42 \pm 6 \%$ of decrement; $P<0.0001$; Fig. 2).

Glucagon responses to insulin-induced hypoglycemia and to stimulation with arginine. Basal glucagon levels at normoglycemia were higher in recipients $(192 \pm 23 \mathrm{ng} / \mathrm{liter})$ than in nonrecipients $(115 \pm 9 \mathrm{ng} / \mathrm{liter} ; P<0.001)$ and controls (113 $\pm 12 \mathrm{ng} /$ liter; $P<0.01$; Fig. 1). During insulin-induced hypoglycemia very little increase in glucagon levels was observed in the nonrecipients. In contrast, glucagon levels increased significantly during hypoglycemia in recipients and in normal control subjects. Maximal incremental glucagon responses to hypoglycemia $(\Delta \max )$ in recipients $(170 \pm 31 \mathrm{ng} /$ liter) were significantly greater $(P<0.0001)$ than nonrecipients ( $37 \pm 7 \mathrm{ng} /$ liter, $P<0.0001)$, and similar to that observed in controls ( $125 \pm 15 \mathrm{ng} /$ liter). In the subset of 12 type I diabetics studied before and after pancreas transplantation, maximal incremental glucagon responses were significantly improved $(52 \pm 15$ vs. $225 \pm 57 \mathrm{ng} /$ liter; $P<0.02)$. Blood glucose recovery correlated significantly with incremental glucagon responses when all recipient data were used $(r=0.49, n=24, P$ $<0.0001$ ), but in the subset of 12 recipients this correlation was not significant $(r=0.38, n=12)$. The effect of beta adrenergic blockade on glucagon secretion during hypoglycemia in three pancreas recipients was examined to ascertain whether improved glucagon responses were related to beta adrenergic input. Glucagon responses were unaffected by an 

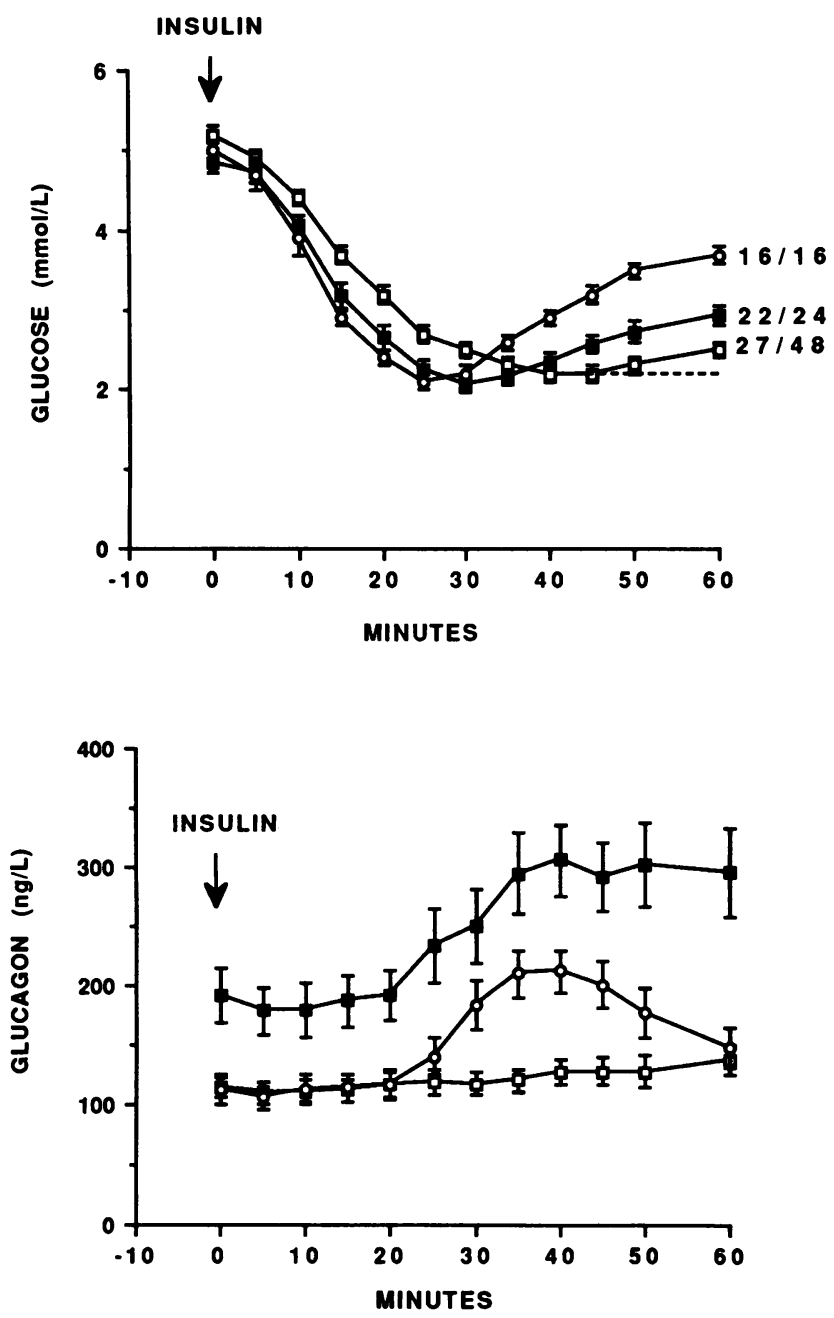

Figure 1. Circulating glucose and glucagon levels before and during insulin-induced hypoglycemia in 24 type I diabetic recipients of pancreas allografts (closed squares), 48 type I diabetic nonrecipients (open squares), and 16 nondiabetic normal control subjects (open circles). The test was completed in 22/24 type I diabetic recipients, $27 / 48$ type I diabetic nonrecipients, and 16/16 normal controls. The dashed line represents an estimation of the theoretical 60-min glucose level that would have been achieved had the $21 / 48$ nonrecipients completed their test. This estimation was made based upon the last three plasma glucose levels before premature discontinuation of this test by injecting intravenous glucose because of severe discomfort. Plasma glucose recovery in recipients of pancreas allografts was significantly improved compared with type I diabetic nonrecipients $(P<0.0001)$, but still significantly lower than that observed in normal control subjects $(P<0.00001)$. Basal levels of glucagon were significantly elevated in pancreas recipients compared with nonrecipients $(P<0.001)$ and controls $(P<0.01)$. The maximal incremental glucagon responses in recipients were significantly greater $(P$ $<0.0001$ ) than nonrecipients and not significantly different than controls.

intravenous infusion of propranolol $(80 \mu \mathrm{g} / \mathrm{min})$ given before and during hypoglycemia (Fig. 3).

Glucagon responses to arginine were also determined in recipients, nonrecipients, and normal control subjects to compare alpha cell responsiveness with direct stimulation. Basal glucagon values were approximately two-fold elevated in recipients compared with nonrecipients and controls (Fig. 4),
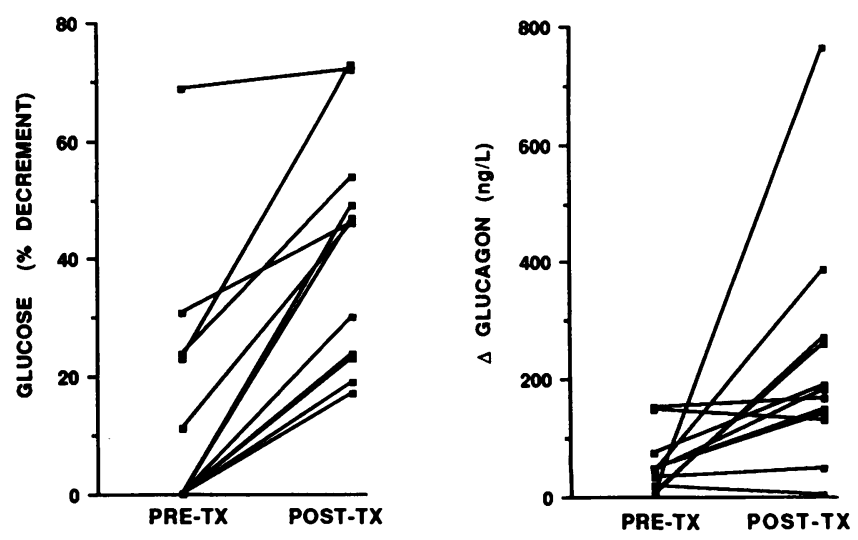

Figure 2. Glucose and glucagon data obtained at $60 \mathrm{~min}$ after the injection of insulin in 12 type I diabetic recipients who were tested before and after pancreas transplantation. Glucose data are expressed as a percentage of the absolute fall in glucose levels (pretest levels minus nadir levels), and glucagon levels are expressed as peak increments over pretest glucagon values. These 12 type I diabetic recipients were tested before and after pancreas transplantation. Differences in plasma glucose recovery at $60 \mathrm{~min}$ are significant at $P$ $<0.0001$, and differences in maximal glucagon increments are significant at $P<0.02$.

and integrated incremental glucagon responses under the curve were significantly greater in recipients $(1910 \pm 207)$ compared with nonrecipients $(710 \pm 70 ; P<0.0001)$ and with controls $(1109 \pm 162 \mathrm{ng} /$ liter $\times$ min, $P<0.001)$.

Catecholamine responses to insulin-induced hypoglycemia. Epinephrine responses to insulin-induced hypoglycemia were highly variable in all three groups (Fig. 5), with a trend towards impaired responses in nonrecipients. However, no significant differences in basal levels or in peak incremental epinephrine responses were observed among nonrecipients $(2.2 \pm 0.3)$, recipients (2.3 \pm 0.4$)$, and controls $(2.6 \pm 0.4 \mathrm{nmol} / \mathrm{liter})$. The results obtained from 6 type I diabetics studied before and after pancreas transplantation also showed no improvement in peak

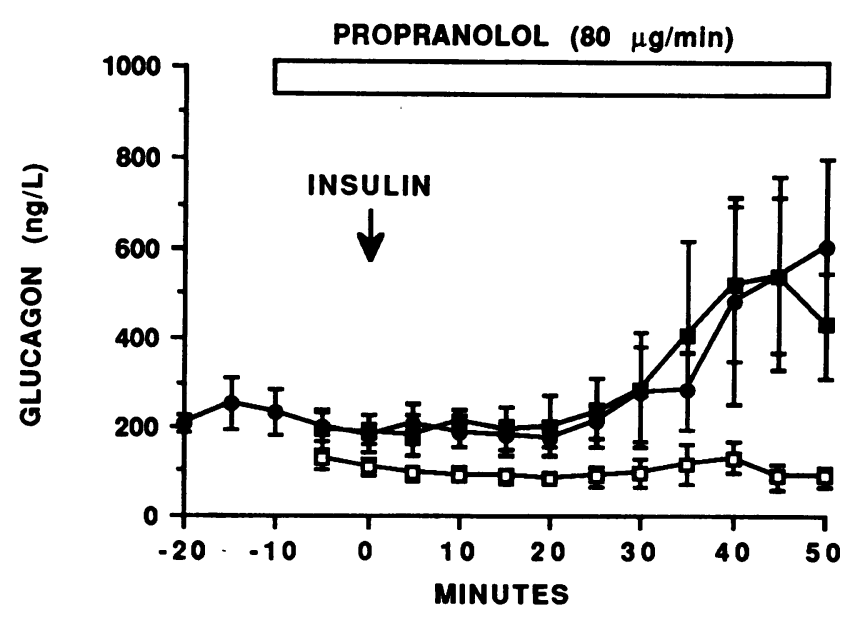

Figure 3. Lack of effect of $\beta$-adenergic blockade (propranolol, $80 \mu \mathrm{g}$ / min i.v. infusion) on plasma glucagon levels during insulin-induced hypoglycemia in three type I diabetic recipients of pancreas allografts (after pancreas transplantation and without propranolol, closed squares; after pancreas transplantation and with propranolol, closed circles; and before pancreas transplantation, open squares). 


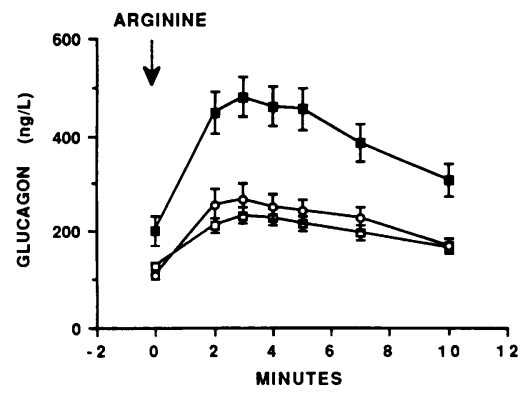

Figure 4. Plasma glucagon levels before and after arginine ( $5 \mathrm{~g}$ i.v.) in 25 type I diabetic recipients of pancreas allografts (closed squares), 46 type I diabetic nonrecipients (open squares), and 18 normal control subjects (open circles). Glucagon secretion expressed as area

under the curve from 0-10 min was significantly greater in type I diabetic pancreas recipients compared with nonrecipients $(P$

$<0.0001)$, and compared with normal control subjects $(P<0.001)$.

incremental epinephrine responses to hypoglycemia $(2.3 \pm 0.6$ $\mathrm{nmol} /$ liter vs. $2.3 \pm 0.6$ ). Similarly, norepinephrine responses showed no differences between the three groups (peak incremental norepinephrine responses: $1.2 \pm 0.2$ in nonrecipients, $n$ $=14 ; 1.5 \pm 0.2$ in recipients, $n=19$; and $1.3 \pm 0.2 \mathrm{nmol} /$ liter in controls, $n=10$ ).

Pancreatic polypeptide responses to hypoglycemia and to secretin stimulation. Basal pancreatic polypeptide levels at normoglycemia did not differ among the three groups $(41 \pm 7$ in nonrecipients, $37 \pm 7$ in recipients, and $24 \pm 6 \mathrm{pmol} / \mathrm{liter}$ in controls; Fig. 6). Peak incremental pancreatic polypeptide responses to hypoglycemia were significantly diminished in nonrecipients $(79 \pm 15 ; P<0.001)$ and in recipients $(70 \pm 15 ; P$ $<0.001)$ compared with control subjects $(195 \pm 37 \mathrm{pmol} / \mathrm{liter})$. During stimulation with intravenous secretin, peak pancreatic polypeptide responses (average of three highest values minus basal value for each subject) in recipients $(67 \pm 24 ; n=17)$, nonrecipients $(45 \pm 19 ; n=25)$, and normal control subjects $(37 \pm 11 \mathrm{pmol} /$ liter; $n=17)$ did not differ.

\section{Discussion}

The studies described herein were designed to determine the effects of successful pancreas transplantation in type I diabetic patients upon the secretion of glucagon, pancreatic polypep-

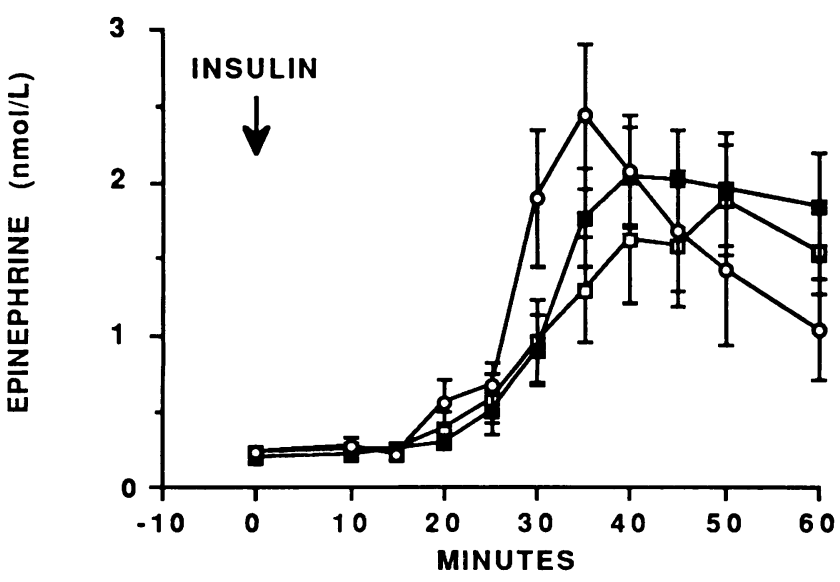

Figure 5. Plasma epinephrine levels before and during insulin tolerance testing in 14 type I diabetic recipients of pancreas allografts (closed squares), 19 type I diabetic nonrecipients (open squares), and 10 normal control subjects (open circles). There were no significant differences among the three groups.

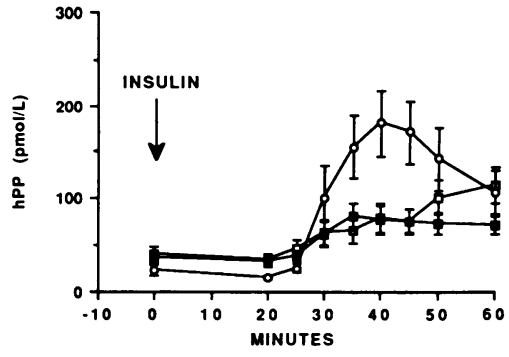

Figure 6. Circulating hPP levels before and during insulin tolerance testing in 24 type I diabetic recipients of pancreas allografts (closed squares), 46 type I diabetic nonrecipients (open squares), and 16 normal controls (open circles). Peak pancreatic polypeptide responses to hypoglycemia were significantly diminished

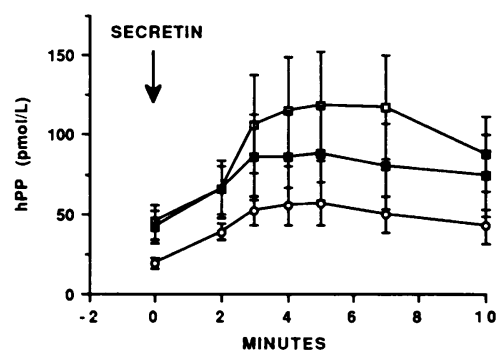
in pancreas recipients $(P<0.001)$ and in nonrecipients $(P$ $<0.001)$ compared with normal control subjects. No differences were observed among the three groups after stimulation with intravenous secretin $(2 \mathrm{U} / \mathrm{kg})$.

tide, and catecholamines. Our data demonstrate significant improvement in, although not normalization of, the magnitude of recovery of circulating glucose levels after insulin-induced hypoglycemia. The improvement in glucose recovery is evident when cross-sectionally comparing the glucose responses of pancreas recipients with those of normal controls and nonrecipients, and when comparing prospectively the glucose responses in the subset of recipients we studied before and after pancreas transplantation. A marked increase in basal and stimulated glucagon secretion was associated with the improved glucose recovery after hypoglycemia in the pancreas recipients. In this subset, 4 of the 12 recipients had no improvement in glucagon responses but two had improved epinephrine responses (in the other two epinephrine data were not obtained), which may explain the improvement in glucose recovery. The reason for the elevated basal glucagon levels is uncertain. Systemic venous drainage of the allograft may be an important factor since glucagon secreted by the allograft would reach the systemic circulation before and not after metabolism by the liver. The liver is known to be an important site of glucagon metabolism with normal hepatic glucagon extraction estimated at $\sim 20-25 \%(18,19)$. The other possible explanations include diminished renal function and steroid therapy. However, Ostman et al. reported that basal glucagon levels in pancreas transplant recipients with systemic venous drainage of their graft were elevated compared with kidney transplant recipients with similar renal function and the same immunosuppressive regimen (20). Regardless of the explanation for elevated basal glucagon levels in our patients, it seems reasonable to contend that the marked increase in glucagon secretion during insulin-induced hypoglycemia is related to the improved glucose recovery after hypoglycemia in our pancreas recipients. It is interesting how this increased glucagon responsivity in recipients is not peculiar to glycopenic signaling, because increased secretion in recipients was also observed during stimulation with arginine. Our data also suggest that islet alpha cells in humans do not require central nervous 
system inputs to respond to glycopenia, although it is generally accepted that central nervous system inputs into the endocrine pancreas participate in islet alpha cell responses to hypoglycemia. The contention that our patients' allografts remained denervated at the time of study seems reasonable since many of our patients were studied by 3 mo after pancreas transplantation. Moreover, even if reinnervation was to occur, it is extremely unlikely that this would establish the normal neural mechanisms inherent in the native pancreas because our patients allografts were positioned in an heterotopic position in the pelvis rather than in the area surrounding the normal pancreatic bed. An alternative interpretation of our data is that return to normoglycemia for an extended period of time may have improved alpha cell function so that hypoglycemia could stimulate secretion from the native pancreas. There are not a great deal of reported data to argue against this possibility, although it has been reported that patients treated with insulin pumps and achieving normoglycemia for a period of up to 12 mo still had defective glucagon responses to hypoglycemia (21, 22). Although there are very few studies of this nature and glucose control with intensive insulin treatment is not as fine as one can obtain through pancreas transplantation, we think it is worth considering that the marked improvement in glucagon secretion in pancreas recipients could be due to the allograft exclusively, since it is well appreciated that alpha cells respond to hypoglycemia in the absence of sympathetic or parasympathetic innervation.

In our study, no significant differences were observed in pancreatic polypeptide responses or catecholamine responses during insulin-induced hypoglycemia when comparing recipients and nonrecipients of pancreas allografts. Interestingly, glucagon responses to arginine, which are not neurally mediated, were greater in the recipients compared with nonrecipients, whereas no significant differences between these two groups were observed in pancreatic polypeptide responses to secretin, which require vagal innervation of the islet $(23,24)$. This further suggests that the transplanted allografts in our patients remained denervated up to the time of our studies. That pancreatic polypeptide secretion is quantitatively similar in recipients and nonrecipients suggests that secretion of this hormone in both groups occurs from native pancreas only. If this reasoning is correct, serial monitoring of pancreatic polypeptide responses may provide a valuable metabolic marker for possible future allograft reinnervation should it occur. Although there appeared to be a trend towards improvement in the epinephrine responses in pancreas recipients, there were no significant differences in the epinephrine responses among the three groups. Regardless, because of this trend and because of the possibility of denervation hypersensitivity to adrenergic stimulation, we wished to establish whether the improved glucagon responses in the recipients might be related to increased adenergic input into pancreatic islet alpha cells through betaadenergic stimulation (25). That propranolol failed to diminish the glucagon responses to hypoglycemia reinforces the possibility that these responses may have been due to direct sensing by the alpha cells of glycopenia rather than concurrent beta-adenergic stimulation from circulating catecholamines.

The effects of pancreas transplantation in humans with type I diabetes mellitus on glucose and hormonal counterregulatory responses to hypoglycemia, and on secretion of glucagon, catecholamines, and pancreatic polypeptide in general, have not been previously reported. The only other report ad- dressing glucagon responsivity after hypoglycemia or other stimulation in pancreas recipients is that of Bosi et al. (26) who reported intact glucagon responses to hypoglycemia in five of six patients who received segmental pancreas allografts. However, this study did not include control data from diabetics or normal subjects for comparison, nor did it provide pretransplantation data from their recipients. Hence, one cannot be certain from their report alone whether segmental transplantation improved glucagon responses or counterregulation of hypoglycemia. The only other relevant report is from Ostman et al. (20) who reported only basal but not stimulated glucagon levels.

The importance of these studies at a pragmatic level lies in the widely recognized observation that absence of glucagon responses to hypoglycemia in patients with type I diabetes mellitus is an important consequence which generally begins to become evident several years after diagnosis of the disease. This leads to deranged counterregulation of hypoglycemia and significant clinical risks. In view of the augmented glucagon responses and improved counterregulation of hypoglycemia demonstrated in our study, it may be reasonable to consider pancreas transplantation in type I diabetic patients who are at proven risk for serious episodes of insulin-induced hypoglycemia and who demonstrate refractoriness to conventional medical management with diet, alteration of insulin dosage, and exogenous glucagon injections.

\section{Acknowledgments}

We are indebted to the nursing and laboratory staff of the University of Minnesota General Clinical Research Center for skilled patient care and excellent technical assistance. In addition we thank Ms. Paula Rossin for help with the preparation of this manuscript.

This work was supported by grants (R01-DK-39994 and M01-RR00400) from the National Institutes of Health and the Medical Research Service of the Department of Veterans Affairs. Dr. Diem was in part supported by a grant from the Swiss National Science Foundation.

\section{References}

1. Sutherland, D. E. R., J. S. Najarian, B. Z. Greenberg, B. J. Senske, G. E. Anderson, R. S. Francis, and F. C. Goetz. 1981. Hormonal and metabolic effects of a pancreatic endocrine graft. Ann. Int. Med. 95:537-541.

2. Pozza, G., J. Traeger, J. M. Dubernard, A. Secchi, A. E. Pontiroli, E. Bosi, M. C. Malik, A. Ruitton, and N. Blanc. 1983. Endocrine responses of type 1 (insulin-dependent) diabetic patients following successful pancreas transplantation. Diabetologia. 24:244-248.

3. Traeger, J., J. M. Dubernard, G. Pozza, E. Bosi, A. Secchi, A. E. Pontiroli, J. L. Touraine, H. Betuel, S. El Yafi, F. Da Ponte, D. Cantarovich, N. Diab, C. Cardozo, X. Martin, G. Kamel, and A. Gelet. 1983. Influence of immunosuppressive therapy on the endocrine function of segmental pancreatic allografts. Transplant. Proc. 15:1326-1329.

4. Pozza, G., E. Bosi, A. Secchi, P. M. Piatti, J. L. Touraine, A. Gelet, A. E. Pontiroli, J. M. Dubernard, and J. Traeger. 1985. Metabolic control of type I (insulin-dependent) diabetes after pancreas transplantation. Brit. Med. J. 291:510-513.

5. Dafoe, D. C., D. A. Campbell, R. M. Merion, L. Rosenberg, L. L. Rocher, A. I. Vinik, A. K. Vine, and J. G. Turcotte. 1987. Pancreatic transplantation-University of Michigan. Transplant. Proc. 19(Suppl):55-62.

6. Land, W., R. Landgraf, W.-D. Illner, D. Abendroth, A. Kampik, U. Jensen, F. P. Lenhart, D. Burg, G. Hillebrand, L. A. Castro, M. M. C. Landgraf-Leurs, L. Frey, M. Gokel, St. Schleibner, J. Nusser, 
and M. Ulbig. 1987. Clinical pancreatic transplantation using the prolamine duct occlusion technique-The Munich experience. Transplant. Proc. 19(Suppl):75-83.

7. Diem, P., M. Abid, J. B. Redmon, D. E. R. Sutherland, and R. P. Robertson. 1990. Systemic venous drainage of pancreas allografts as independent cause of hyperinsulinemia in type I diabetic recipients. Diabetes. 39:534-540.

8. Gerich, J. E. 1988. Glucose counterregulation and its impact on diabetes mellitus. Diabetes. 37:1608-1617.

9. Gerich, J. E., M. Langlois, O. Noacco, J. H. Karam, and P. H. Forsham. 1979. Lack of glucagon response to hypoglycemia in diabetes: evidence for an intrinsic pancreatic alpha cell defect. Science (Wash. DC). 182:171-173.

10. Campbell, L. V., E. W. Kraegen, H. Meler, and L. Lazarus. 1979. Hormonal responses to insulin infusion in diabetes mellitus. Diabetologia. 16:359-364.

11. Boden, G., G. A. Reichard, R. D. Hoeldtke, I. Rezvani, and O. E. Owen. 1981. Severe insulin induced hypoglycemia associated with deficiencies in the release of counterregulatory hormones. $N$. Engl. J. Med. 305:1200-1205.

12. Benson, J. W. Jr., D. G. Johnson, J. P. Palmer, P. L. Werner, and J. W. Ensinck. 1977. Glucagon and catecholamine secretion during hypoglycemia in normal diabetic man. J. Clin. Endocrinol. Metab. 44:459-464.

13. Maher, T. D., R. J. Taneberg, B. Z. Greenberg, J. E. Hoffman, R. P. Doe, and F. C. Goetz. 1977. Lack of glucagon secretion during hypoglycemia in diabetic autonomic neuropathy. Diabetes. 26:196200.

14. Sutherland, D. E. R., D. L. Dunn, F. C. Goetz, W. Kennedy, R. C. Ramsay, M. W. Steffes, S. M. Mauer, R. Gruessner, K. C Moudry-Munns, P. Morel, A. Viste, R. P. Robertson, and J. S. Najarian. 1989. A 10-year experience with 290 pancreas transplants at a single institution. Ann. Surg. 210:274-288.

15. Harris, V., G. R. Faloona, and R. H. Unger. 1979. Glucagon. In Methods of Hormone Radioimmunoassay. B. M. Jaffe and H. R. Behrman, editors. Academic Press, New York. 643.

16. Chance, R. E., N. E. Moon, and M. G. Johnson. 1979. Human pancreatic polypeptide (HPP) and bovine pancreatic polypeptide
(BPP). In Methods of Hormone Radioimmunoassay. B. M. Jaffe and H. R. Behrman, editors. Academic Press, New York. 657-672.

17. Evans, M. I., J. B. Halter, and D. Porte, Jr. 1978. Comparison of double and single isotope enzymatic derivative methods for measurement of catecholamines in human plasma. Clin. Chem. 24:567570 .

18. Marco, J., J. Diego, M. Villanueva, M. Dray-Fierros, I. Valverde, and J. Segovia. 1973. Elevated plasma glucagon levels in cirrhosis of the liver. N. Engl. J. Med. 289:1107-1111.

19. Jaspan, J. B., K. S. Polonsky, M. Lewis, J. Pensler, W. Pugh, A. R. Moossa, and A. H. Rubenstein. 1981. Hepatic metabolism of glucagon in the dog. Am. J. Physiol. 240:E233-244.

20. Ostman, J., J. Bolinder, R. Gunnarson, C. Brattstrom, G. Tyden, J. Wahren, and C.-G. Groth. 1989. Effects of pancreas transplantation on metabolic and hormonal profiles in IDDM patients. Diabetes. 38(Suppl. 1):88-93.

21. Bergenstal, R. M., K. S. Polonsky, G. Pons, J. B. Jaspan, and A. H. Rubenstein. 1983. Lack of glucagon response to hypoglycemia in type I diabetics after long-term optimal therapy with a continuous subcutaneous insulin infusion pump. Diabetes. 32:398-402.

22. Bolli, G., G. Calabrese, P. De Feo, P. Compagnucci, G. Zegra, G. Angeletti, M. G. Cartechini, F. Santeusanio, and P. Brunetti. 1982. Lack of glucagon response in glucose counterregulation in type I (insulin-dependent) diabetics: absence of recovery after prolonged optimal insulin therapy. Diabetologia. 22:100-105.

23. Schwartz, T. W. 1983. Pancreatic polypeptide: a hormone under vagal control. Gastroenterology. 84:1411-1425.

24. Glaser, B., A. I. Vinik, A. A. Sive, and J. C. Floyd, Jr. 1980. Plasma human pancreatic polypeptide responses to administered secretin: effects of surgical vagotomy, cholinergic blockade, and chronic pancreatitis. J. Clin. Endocrinol. \& Metab. 50:1094-9.

25. Gerich, J. E., M. Lorenzi, E. Tsalikian, and J. H. Karam. 1976. Studies on the mechanism of epinephrine-induced hyperglycemia in man. Diabetes. 25:65-71.

26. Bosi, E., P. M. Piatti, A. Secchi, L. D. Monti, J. Traeger, J. M. Dubernard, and G. Pozza. 1988. Response of glucagon and insulin secretion to insulin-induced hypoglycemia in type I diabetic recipients after pancreatic transplantation. Diab. Nutr. Metab. 1:21-27. 